Review

\title{
VLBI: A fascinating technique for geodesy and astrometry
}

\author{
H. Schuha,*, D. Behrend ${ }^{\mathrm{b}}$ \\ a Institute of Geodesy and Geophysics, University of Technology, Vienna, Austria \\ ${ }^{\mathrm{b}}$ NVI, Inc./NASA Goddard Space Flight Center, Code 698.2, Greenbelt, MD, USA
}

\section{A R T I C L E I N F O}

\section{Article history:}

Received 30 January 2012

Received in revised form 10 July 2012

Accepted 30 July 2012

Available online 10 August 2012

\section{Keywords:}

Very Long Baseline Interferometry (VLBI)

IVS

VLBI2010

CRF

TRF

Earth orientation

Global Geodetic Observing System (GGOS)

\begin{abstract}
A B S T R A C T
Since the 1970s Very Long Baseline Interferometry (VLBI) has proven to be a primary space-geodetic technique by determining precise coordinates on the Earth, by monitoring the variable Earth rotation and orientation with highest precision, and by deriving many other parameters of the Earth system. VLBI provides an important linkage to astronomy through, for instance, the determination of very precise coordinates of extragalactic radio sources. Additionally, it contributes to determining parameters of relativistic and cosmological models. After a short review of the history of geodetic VLBI and a summary of recent results, this paper describes future perspectives of this fascinating technique. The International VLBI Service for Geodesy and Astrometry (IVS), as a service of the International Association of Geodesy (IAG) and the International Astronomical Union (IAU), is well on its way to fully defining a next generation VLBI system, called VLBI2010. The goals of the new system are to achieve on scales up to the size of the Earth an accuracy of $1 \mathrm{~mm}$ in position and of $0.1 \mathrm{~mm} /$ year in velocity. Continuous observations shall be carried out $24 \mathrm{~h}$ per day 7 days per week in the future with initial results to be delivered within $24 \mathrm{~h}$ after taking the data. Special sessions, e.g. for monitoring the Earth rotation parameters, will provide the results in near real-time. These goals require a completely new technical and conceptual design of VLBI measurements. Based on extensive simulation studies, strategies have been developed by the IVS to significantly improve its product accuracy through the use of a network of small ( $\sim 12 \mathrm{~m}$ ) fast-slewing antennas. A new method for generating high precision delay measurements as well as improved methods for handling biases related to radio source structure, system electronics, and deformations of the antenna structures has been developed. Furthermore, as of January 2012, the construction of ten new VLBI2010 sites has been funded, with good prospects for one dozen more antennas, which will improve the geographical distribution of geodetic VLBI sites on Earth and provide an important step toward a global VLBI2010 network. Within this paper, the Global Geodetic Observing System (GGOS) of the IAG will also be introduced and the contribution of VLBI to GGOS will be described.
\end{abstract}

(c) 2012 Published by Elsevier Ltd.

\section{Contents}

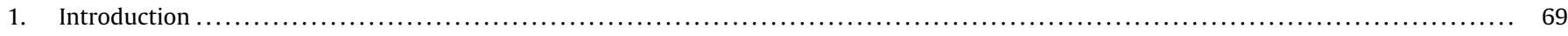

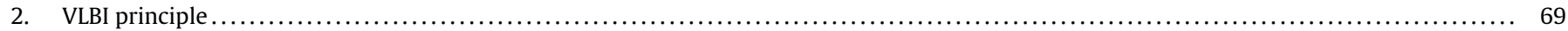

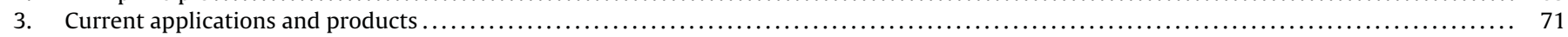

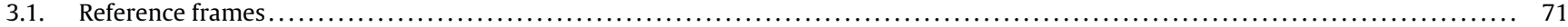

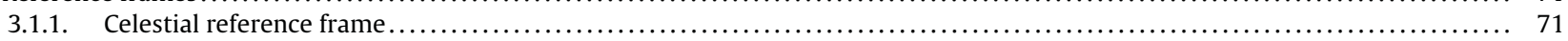

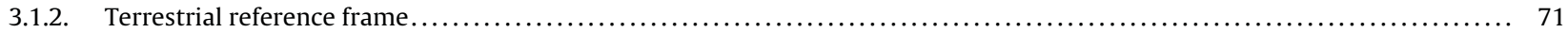

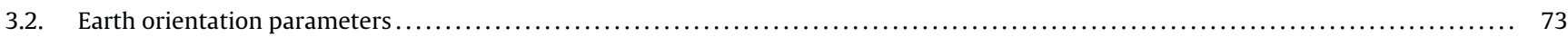

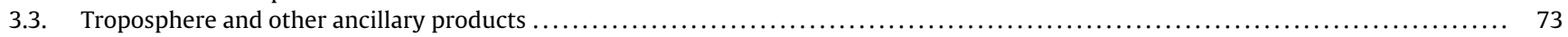

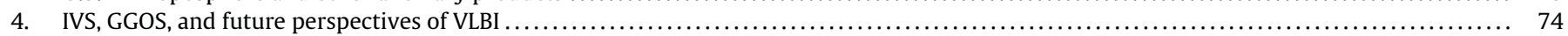

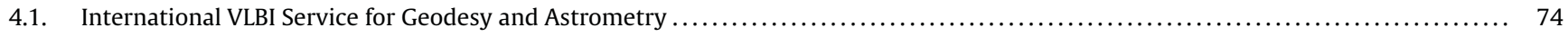

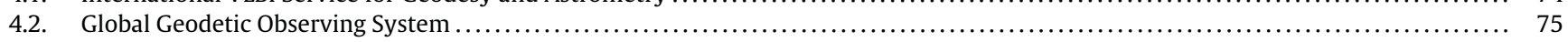

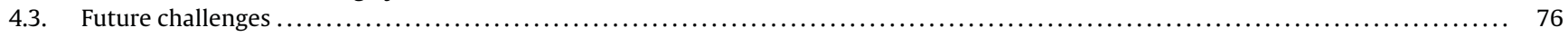

\footnotetext{
* Corresponding author. 


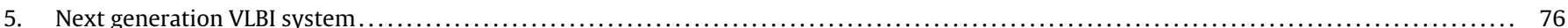

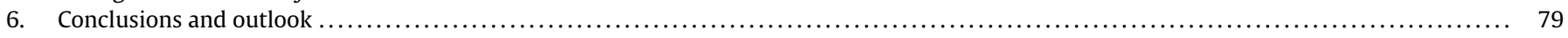

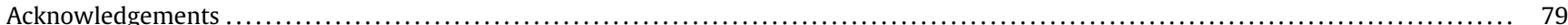

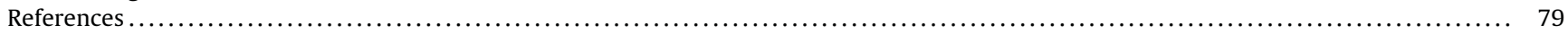

\section{Introduction}

The technique that has come to be known as Very Long Baseline Interferometry (VLBI) dates back more than forty years to the mid-1960s. Initially conceived as a radio astronomy tool to investigate objects in the sky, its potential for geodetic applications was recognized early on. In 1969 work was already done in geodesy, astrometry, and clock synchronization, yielding accuracies of 2-5 m and in radio source position on the order of $1^{\prime \prime}$ (Sovers et al., 1998). Early VLBI measurements were hindered for geodetic accuracy by several factors. Firstly, they were generally conducted using rubidium oscillators, which did not have the necessary stability for good geodetic measurements. Secondly, recorded bandwidths were too small to obtain good group delay measurements (see Section 2). Still, the geodetic VLBI developments and observations of 1969 laid the groundwork for the modern multi-band and broadband geodetic VLBI techniques that are used today and that are many times more accurate. From the early start the technical evolution of geodetic and astrometric VLBI has been fast-paced. Experiments in 1972 with the Mark I system obtained a formal error for the baseline length of $\sim 70 \mathrm{~mm}$, with the accuracy being in the range of a few decimeters; by the end of the century, the formal error was under $1 \mathrm{~mm}$ for the best global baselines, with accuracies around 5-10 mm. Hence, in a quarter-century the VLBI formal error as well as the (more realistic) accuracy improved by a factor of nearly 100 ; most of that was achieved in the early 1980s by introducing the so-called bandwidth synthesis method to increase the recorded effective bandwidth (see, e.g., Ryan and Ma, 1998). The state-of-theart of the VLBI techniques at the end of the last century along with its historical development is described in a number of review papers. The interested reader is referred to Robertson (1991), Sovers et al. (1998), or Ryan and Ma (1998) and further references therein.

In the first decade of the new century geodetic and astrometric VLBI has been further refined in terms of technology and organization. Worth mentioning for the former are the change from tape to disk recording, the gradual transition from hardware to software correlation, and recording at higher data rates. These improvements have been applied to the currently used S/X VLBI system (legacy system), which performs measurements at $\mathrm{S}$ band $(2.2-2.4 \mathrm{GHz})$ and $\mathrm{X}$ band $(8.2-8.95 \mathrm{GHz})$. A next generation VLBI system based on broadband delay is under development, sometimes referred to as the VLBI2010 system, and is expected to become operational in the next several years.

On the organizational side, we saw the establishment of the International VLBI Service for Geodesy and Astrometry (IVS) and the creation of the Global Geodetic Observing System (GGOS). Prior to the existence of the IVS, most of the scientific and operational activities in geodetic and astrometric VLBI were organized through national or bi-lateral agreements, sometimes on an ad hoc basis (Schlüter and Behrend, 2007). Today the global VLBI resources and observing plans are coordinated by the IVS. The IVS, in turn, is an integral part of the GGOS of the International Association of Geodesy (IAG) as well as a service of the International Astronomical Union (IAU); thus, VLBI plays an indispensable role in modern science as demonstrated in the simplified scheme depicted in Fig. 1.

In this paper we describe the current applications and products obtained with the S/X VLBI system. We then present the GGOS and future challenges for Earth sciences in general and for VLBI in particular. This leads us to an overview of the development of the next generation VLBI system, VLBI2010.

\section{VLBI principle}

The idea that underlies geodetic VLBI observations is conceptually quite simple: the fundamental observable is the difference in arrival times (time delay) of a signal from an extragalactic radio

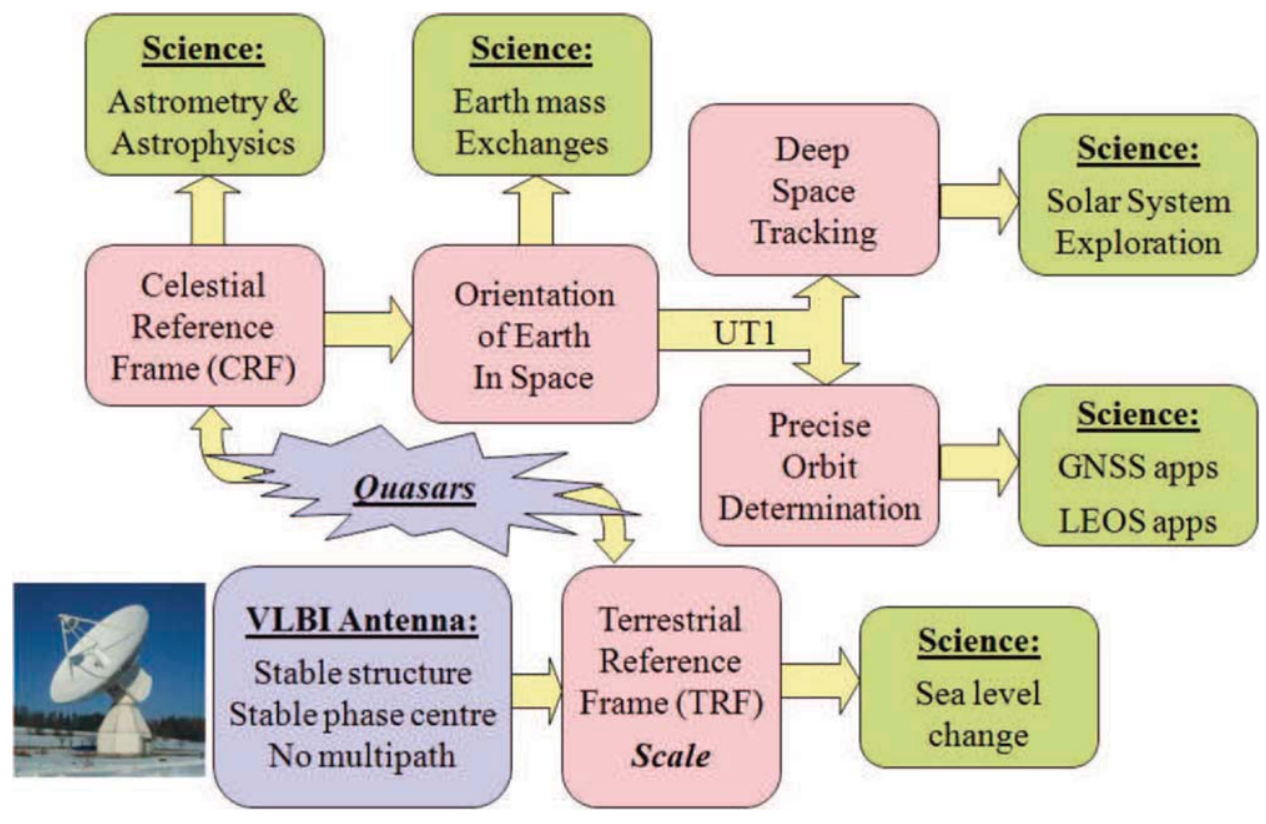

Fig. 1. Simplified scheme of the roles of VLBI in science. 


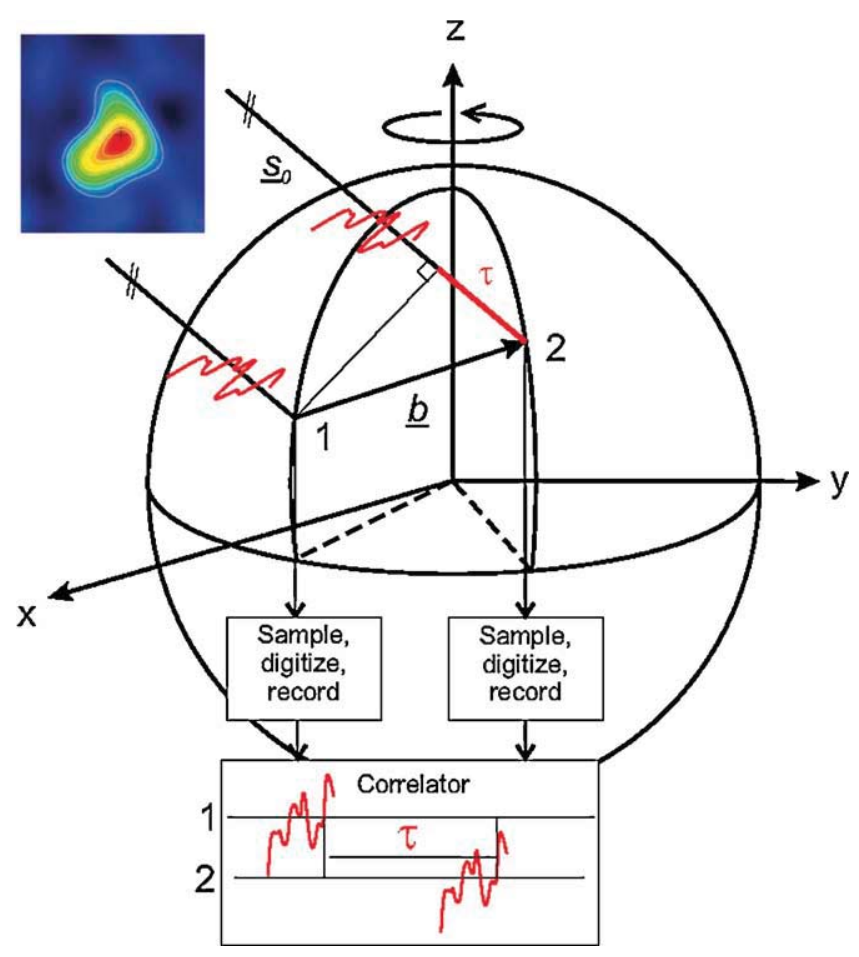

Fig. 2. Basic concept of Very Long Baseline Interferometry. Courtesy of J. Böhm.

source received at two (or more) radio observatories (Robertson, 1991). This observable only depends on fundamental physics as it is derived from a realization of the atomic second and a clock synchronization convention. The time delay $\tau$ is measured by amplifying, down-converting in frequency, and digitally sampling, the radiation received from the quasar at each antenna site with electronic signal processing devices whose time, frequency, and phase information is derived coherently from an on-site atomic frequency standard, usually a hydrogen maser (Cannon, 1999).

As shown in Fig. 2, the basic concept of VLBI consists of an incoming planar wave front that propagates along the unit vector to the radio source $\mathbf{s}_{0}$ and arrives at two antennas, which point simultaneously at the same radio source and are separated by the baseline vector $\mathbf{b}$. The scalar product of $\mathbf{b}$ and $\mathbf{s}_{0}$ divided by the speed of light $c$ determines the time delay (geometric delay) $\tau_{g}$ :

$\tau_{g}=-\frac{\mathbf{b} \cdot \mathbf{s}_{0}}{c}=t_{2}-t_{1}$

Repeated determinations of the geometric delay $\tau_{g}$ for many radio sources with sequential observations made in rapid succession, results in a data set sufficient to over-determine the baseline vector b and/or the coordinates of the observed radio sources.

Along with precise station time information, the sampled quasar radiation is digitally recorded and sent to a special purpose VLBI correlator facility for cross-correlation processing. The crosscorrelation determines the time lag between the two bitstreams by finding when they match best. This process requires that over the integration period the effects of Earth rotation on the station positions be corrected; for instance, the additional path length that the planar wave front has to travel after hitting point 1 and prior to reaching point 2 has to be accounted for as the Earth (and thus point 2) has moved in between the two events. The relative delay, at which the peak of the cross-correlation function is found, yields a value of $\tau$ (Cannon, 1999).

As in all correlation functions, the width $w$ of the central maximum of the VLBI cross-correlation function is inversely related to the bandwidth of the signals being cross-correlated $(w \propto 1 / \Delta v)$. The precision with which the value of the signal delay $\tau$ may be measured is given by $\sigma_{\tau}$ and is related to the effective bandwidth $B_{\text {eff }}$ of the cross-correlated signal by

$\sigma_{\tau}=\frac{1}{2 \pi} \cdot \frac{1}{\mathrm{SNR} \cdot B_{\text {eff }}}$

where SNR designates the signal-to-noise ratio observed on the interference fringes and $B_{\text {eff }}$ refers to the effective bandwidth of the recorded VLBI signals (Cannon, 1999).

The VLBI signal delay $\tau$ measured at the correlator is dominated by the geometric delay $\tau_{g}$, but also contains other significant contributions that require attention. Adding correction terms to the geometrical delay yields the fundamental observation equation for VLBI (Cannon, 1999):

$\tau=\tau_{g}+\tau_{a b}+\tau_{c l k}+\tau_{\text {inst }}+\tau_{\text {trop }}+\tau_{\text {iono }}+\tau_{\text {rel }}$

where

- $\tau_{g}$ is the geometric delay;

- $\tau_{a b}$ is a contribution due to diurnal abberation;

- $\tau_{c l k}$ is a contribution to the signal delay arising from the missynchronization of the reference clocks at each observatory;

- $\tau_{\text {inst }}$ is a contribution to the signal delay arising from the propagation delays through on-site cable runs and other instrumentation;

- $\tau_{\text {trop }}$ is a contribution to the signal delay arising from the propagation delays through the non-ionized portions of the Earth's atmosphere;

- $\tau_{\text {iono }}$ is a contribution to the signal delay arising from the propagation delays through the ionized portions of the Earth's atmosphere;

- $\tau_{\text {rel }}$ are special and general relativistic corrections to the classical geometric delay $\tau_{g}$.

From the point-of-view of geodetic VLBI, all these terms, with the exception of $\tau_{\text {rel }}$, are small nuisance terms that corrupt the geometric delay $\tau_{g}$. The effects of all of these terms, including $\tau_{r e l}$, must be accounted for and then removed by one of several methods (Cannon, 1999):

- computation from known physics $\left(\tau_{a b}, \tau_{r e l}\right)$;

- calibration $\left(\tau_{\text {inst }}\right)$;

- least squares estimation by modeling, possibly with the aid of locally measured input parameters $\left(\tau_{c l k}, \tau_{\text {trop }}\right)$;

- removal based on dual-frequency observations $\left(\tau_{\text {iono }}\right)$.

The above given delay model represents a good approximation. In a rigorous approach, however, the modeling needs to be fully done within relativistic spacetime. The interested reader is referred to corresponding publications by Finkelstein et al. (1983) and Müller (1991) for more details.

In VLBI literature the observable $\tau$ is often referred to as group delay. This is the primary observable currently used in geodetic and astrometric VLBI. In practice, the group delay $\tau_{g d}=\tau$ is determined in the correlation process by fitting a straight line to a sequence of phases measured at several discrete frequencies. When $\phi$ is the fringe phase and $\omega=2 \pi \nu$ is the observed angular frequency, the group delay can be written as

$\tau_{g d}=\frac{\partial \phi}{\partial \omega}$ 
The group delay thus is the slope of phase with respect to frequency. A potentially very precise observable is the phase delay, which is obtained from the quotient of phase and frequency:

$\tau_{p d}=\frac{\phi}{\omega}$.

However, the interpretation of the phase requires the determination of the number of unknown phase cycles (ambiguity problem). If the $a$ priori geometry (antenna positions, radio source positions, and propagation delays) is not known to better than a wavelength, the count of the number of wavelengths can easily be lost. With enough phase delay measurements, one may be able to bootstrap to the correct solution by trying out various ambiguity sets. However, with increasing length this bootstrap process becomes more and more difficult, as each measurement may have a different ambiguity (Shaffer, 1995). For the next generation VLBI system (cf. Section 5), a four-band system is under development, which is anticipated to measure four tunable frequency bands within the entire range from 2 to $14 \mathrm{GHz}$ allowing the use of the phase delay in the so-called broadband delay.

\section{Current applications and products}

Under the umbrella of the IVS (cf. Section 4), geodetic and astrometric VLBI is being applied to furnish a number of products. These are provided on a regular basis and stem from a continuous monitoring program. The main products and their features are listed in Table 1 . In addition, ancillary products are provided on an irregular basis. In the following we describe the main products in more detail using recent examples. Then we present selected ancillary products.

\subsection{Reference frames}

In geodesy we distinguish by convention between reference systems and reference frames. A reference system is the complete conceptual definition of how a coordinate system is formed, including origin and orientation as well as the underlying fundamental mathematical and physical models (Seeber, 2003). A reference frame is a practical realization of a reference system through observations and consists of a set of identifiable fiducial points on the sky (e.g., radio sources) or on the Earth's surface (e.g., fundamental stations). It is described by a catalog of precise positions and motions at a specific epoch (Seeber, 2003).

The VLBI technique is instrumental in establishing and maintaining the conventional celestial reference frame, called the International Celestial Reference Frame (ICRF). The first realization of the ICRF in 1998 replaced the optical Fundamental Catalogue (FK6 being its last realization). While VLBI is the sole technique to contribute to the ICRF, the International Terrestrial Reference Frame (ITRF), which is the conventional terrestrial reference frame, is assembled by combining the results from several space-geodetic techniques. The combination procedure takes advantage of the strengths of each individual technique and reduces the impact of systematic errors as much as possible. VLBI contributes significantly to the scale of the ITRF as the extremely precise delay measurements done in the time domain are converted to metric distances just by applying the velocity of light-one of the fundamental natural constants.

\subsubsection{Celestial reference frame}

The conceptual basis of reference frames defined by extragalactic objects is straightforward: the universe as a whole does not rotate, hence very distant objects cannot have an overall rotational motion. Experimentally, the global rotation of the universe is less than $10^{-12}$ arcseconds/year as inferred from the $3 \mathrm{~K}$ microwave

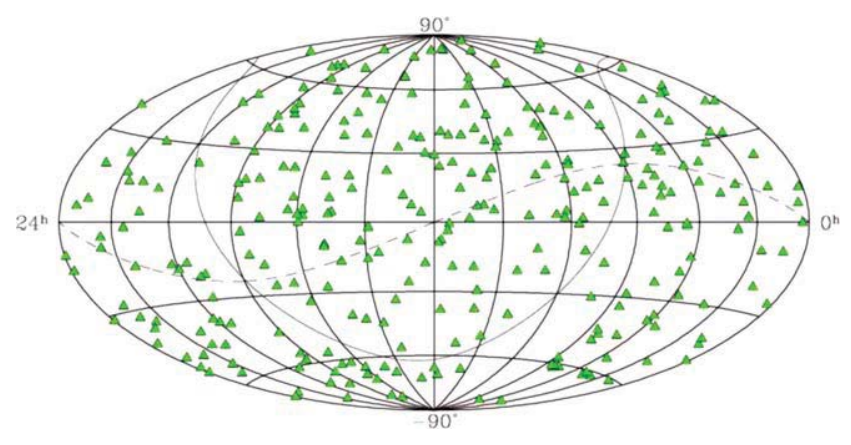

Fig. 3. A sky map of the 295 defining radio sources of ICRF2 based on VLBI observations.

background radiation (Ma, 1999). At a distance of 108 parsecs, the angular velocity of an object is less than $0.6 \times 10^{-3}$ arcseconds/year, while an object moving at a physically more reasonable speed comparable to the Sun would move with $10^{-6}$ arcseconds/year, entirely undetectable by current technology (Ma, 1999).

The analysis of VLBI data for astrometric and geodetic purposes requires the estimation of source positions, station positions, Earth orientation parameters (EOP, cf. Section 3.2), and parameters characterizing the behavior of the clock and atmosphere at each station as well as careful modeling of all geophysical and astronomical effects (cf. Section 2). Each separate VLBI session provides the relative positions of the observed radio sources with some uncertainty. On different days different sets of sources are observed. It is the overlap of some common sources from one day to another that connects all the positions together. The first realization of the ICRF was adopted by the International Astronomical Union (IAU) effective 1 January 1998. It consisted of 608 extragalactic radio sources, 212 of which were defining sources (Ma, 1999). The original ICRF was extended and improved two times using newer data (ICRFExtension.1 and ICRF-Extension.2) to finally encompass some 700 radio source positions.

A decade after the analysis for the first realization, it became evident that much better astrometric and source structure data as well as refined geophysical models were in hand in order to justify the computation of a second realization (Ma, 2010). The Second Realization of the International Celestial Reference Frame (ICRF2) was adopted at the XXVII IAU General Assembly in Rio de Janeiro, Brazil as Resolution B3. The ICRF2 replaced the previously used first realization (ICRF) effective 1 January 2010. The International Earth Rotation and Reference Systems Service (IERS) published Technical Note \#35 (Fey et al., 2009) about the computation of the ICRF2; the generation of the ICRF2 was a common effort of a combined working group of the IERS and the IVS. ICRF2 contains precise positions of 3414 compact extragalactic radio sources, more than five times the number in the ICRF, 295 of which are defining sources known with highest precision (see Fig. 3). Further, the ICRF2 is found to have a noise floor of $\sim 40 \mu$ as, some five to six times better than ICRF, and an axis stability of $\sim 10 \mu$ as, nearly twice as stable as ICRF. Alignment of ICRF2 with the International Celestial Reference System (ICRS) was made using 138 stable sources common to both ICRF2 and ICRF-Ext2.

\subsubsection{Terrestrial reference frame}

Unlike the ICRF, the realization of the International Terrestrial Reference System (ITRS) is a combination of four contributing space-geodetic techniques. The latest version is the International Terrestrial Reference Frame 2008 (ITRF2008), which is based on input data from 1980 through 2008 inclusively. The time series consisted of 29 years of VLBI data, 26 years of SLR data, 12.5 years of GPS data, and 16 years of DORIS data (Altamimi et al., 2011). The 
Table 1

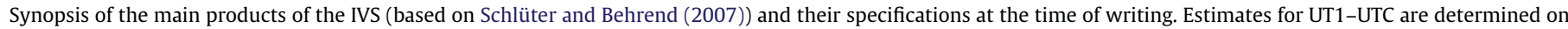
the basis of the 24-h sessions as part of the full set of five EOP parameters and on the basis of the 1-h Intensive sessions for UT1 prediction purposes.

\begin{tabular}{|c|c|c|c|c|c|c|}
\hline & Polar motion $\left(x_{p}, y_{p}\right)$ & UT1-UTC 24-h session & UT1-UTC intensive & Celestial pole $(d X, d Y)$ & $\operatorname{TRF}(x, y, z)$ & $\operatorname{CRF}(\alpha, \delta)$ \\
\hline Accuracy & $50-80 \mu$ as & $3-5 \mu \mathrm{s}$ & $15-20 \mu \mathrm{s}$ & $50 \mu$ as & $5 \mathrm{~mm}$ & $40-250 \mu$ as \\
\hline Product delivery & $8-10$ days & $8-10$ days & 1 day & $8-10$ days & - & 3 months \\
\hline Resolution & 1 day & 1 day & 1 day & 1 day & - & - \\
\hline Frequency of solution & $\sim 3$ days/week & 〜3 days/week & daily & $\sim 3$ days/week & - & 1 year \\
\hline
\end{tabular}

Table 2

WRMS consistency of the individual VLBI solutions on the basis of horizontal and vertical position, polar motion, and UT1 parameters (based on Böckmann et al. (2010)).

\begin{tabular}{ll}
\hline & Consistency \\
\hline Horizontal position & $1 \mathrm{~mm}$ WRMS \\
Vertical position & $2 \mathrm{~mm}$ WRMS \\
Polar motion & $50 \mu$ as WRMS \\
UT1 & $3 \mu \mathrm{s}$ WRMS \\
\hline
\end{tabular}

ITRF2008 network comprises 934 stations located at 580 sites, with 463 sites in the northern hemisphere and 117 in the southern hemisphere. As shown in Fig. 4, the ITRF2008 combination involved 84 co-location sites where two or more technique instruments were operating at the same site and for which local ties were available (Altamimi et al., 2011).

The VLBI contribution, which was organized under the auspices of the IVS, was a combination of seven individual solutions in the form of session-wise datum-free normal equations. The total number of 24-h sessions that flowed into the overall solution amounted to 4539 covering the time period from 1979.7 to 2009.0 and including data from 115 different VLBI sites (Böckmann et al., 2010). About half of these VLBI sites were occupied only a few times with mobile VLBI systems; several other VLBI stations are mainly used for astronomic purposes and thus cannot be considered as primary geodetic sites. The precision of the overall solution could be determined from the consistency of the individual VLBI solutions expressed in weighted root mean square (WRMS) values (see Table 2).

The datum (origin, orientation, and scale) of ITRF2008 was realized through aligning its axes to the previously used ITRF2005. The ITRF2008 origin was defined using SLR data so that there were no translations and no translation rates w.r.t. the mean center of mass of the Earth. The scale was chosen to be the mean of the stacked VLBI and SLR time series so that the scale factor and its scale rate vanished with respect to this mean. Altamimi et al. (2011) estimated the accuracy of the origin and scale of ITRF2008 to be at the cmlevel, with the scale exhibiting a consistency of about $1.2 \mathrm{ppb}$ (parts

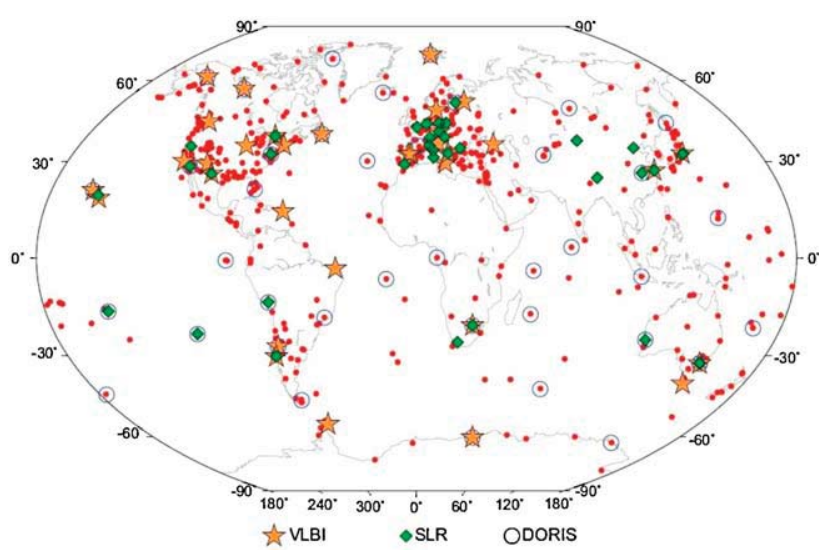

Fig. 4. ITRF2008 network highlighting VLBI, SLR, and DORIS sites co-located with GPS.

From Altamimi et al. (2011), Fig. 1. per billion) which corresponds to roughly $8 \mathrm{~mm}$ at the equator. They envisioned further advancements in the ITRF by improving the consistency of the local ties at co-location sites and by mitigating technique-specific systematic errors. Typical VLBI-specific results w.r.t. the ITRF are, for instance, VLBI-derived horizontal station velocities (Fig. 5) and the co-seismic displacement of TIGO Concepción due to the M8.8 earthquake in 2010 as determined by VLBI (Fig. 6). A typical intercontinental baseline (Wettzell, Germany to Westford, MA, USA) with an observing history of close to 30 years is shown in Fig. 7. The baseline length increases by about $16.9 \pm 0.03 \mathrm{~mm} /$ year due to continental plate motion.

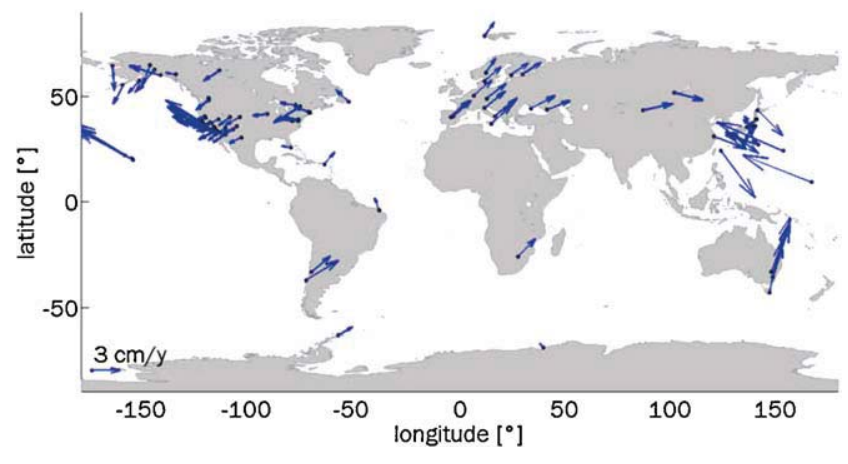

Fig. 5. VLBI-derived horizontal station velocities in the ITRF2008 frame. Courtesy of H. Krásná.
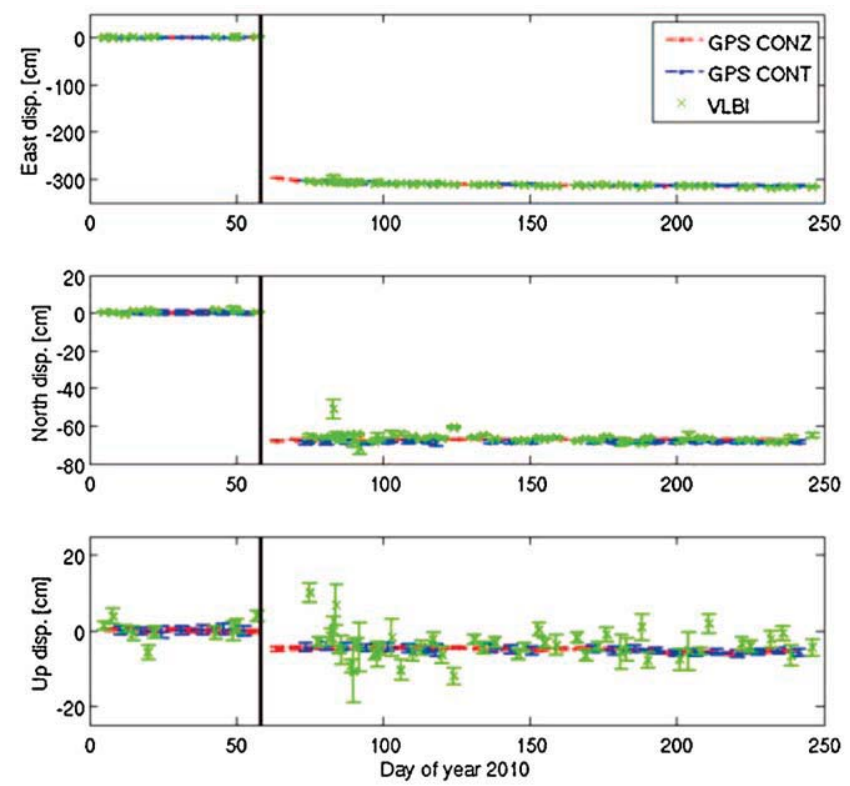

Fig. 6. Co-seismic displacement of TIGO Concepción due to the M8.8 earthquake in 2010 as determined by VLBI and GPS. The scatter and error bars for VLBI are larger, since these are 24-h data as opposed to weekly means used with GPS. The site moved about $3 \mathrm{~m}$ to the west/south-west. 


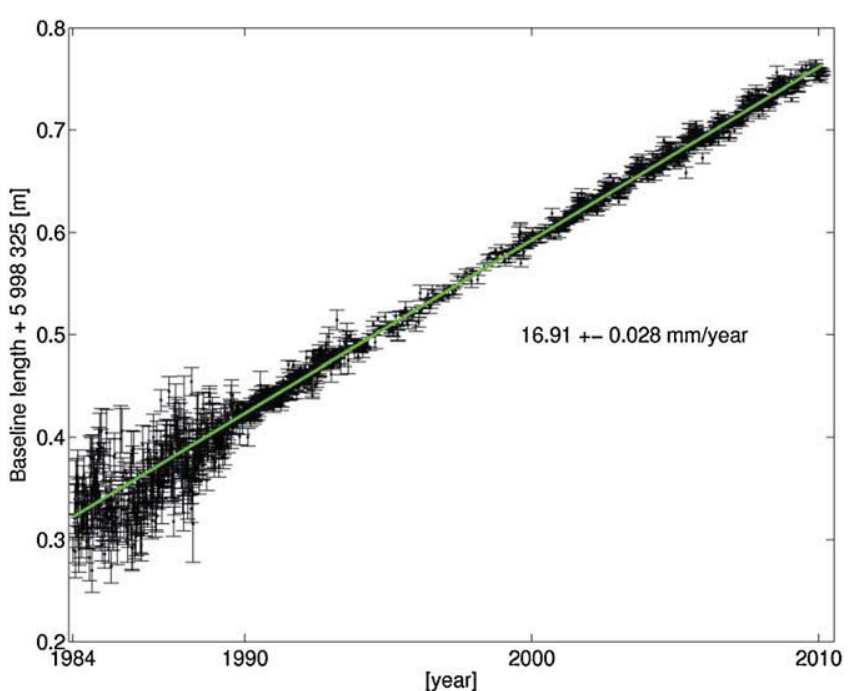

Fig. 7. Baseline length between the stations at Wettzell, Germany and Westford, MA, USA as observed by VLBI from 1984 to 2010.

\subsection{Earth orientation parameters}

The transition from the conventional inertial system to the conventional terrestrial system is realized through a sequence of rotations that account for precession/nutation, Earth rotation, and polar motion. The complete set of these rotations is commonly referred to as Earth orientation parameters (EOP). The subset of Earth rotation and polar motion is sometimes referred to as Earth rotation parameters (ERP). However, this dividing line is somewhat blurred, as in the literature EOP and ERP are often used interchangeably.

Geodetic VLBI is the only space-geodetic technique that allows the observation of the full set of EOP, and it is unique in providing Universal Time (UT1) (see Fig. 8) as well as celestial pole offsets (precession/nutation) over longer time spans. In Fig. 8, the UT1-UTC residuals of the individual Analysis Center solutions are plotted w.r.t. the weighted mean that represents the official solution of the IVS (cf. Section 4). The combination technique furnishes a $20-30 \%$ improvement in robustness and accuracy compared to the submission of the individual Analysis Centers. Fig. 9 depicts polar motion estimates as determined from VLBI observations since 1984 using the Vienna VLBI software VieVS (Nilsson et al., 2011). In Fig. 10 the observed celestial pole offsets $(d X, d Y)$ are plotted w.r.t. the IAU 2000/2006 nutation/precession model.

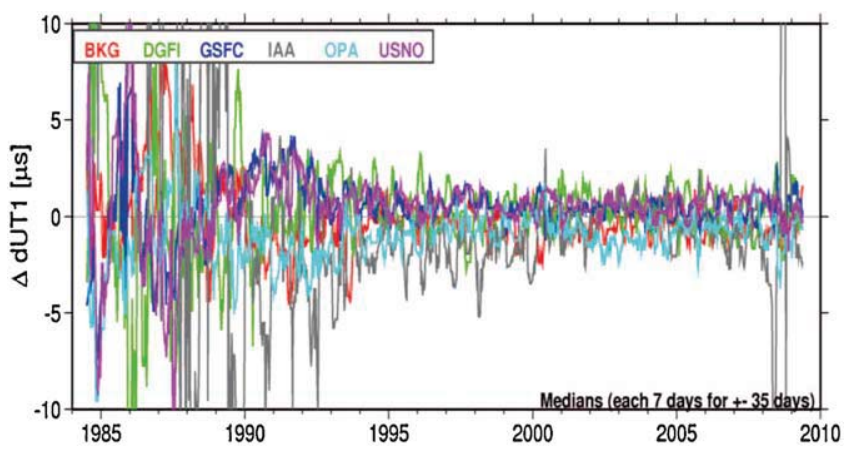

Fig. 8. UT1-UTC residuals based on the combination solution from six IVS Analysis Centers. The combination technique furnishes a $20-30 \%$ improvement in robustness and accuracy as compared to the individual solutions of the Analysis Centers.

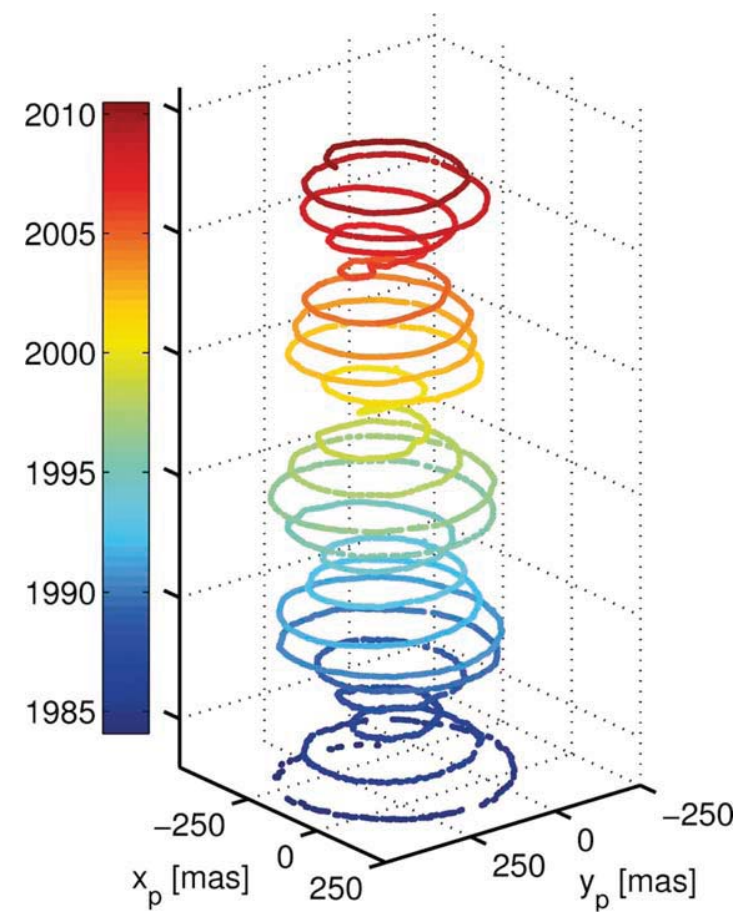

Fig. 9. Color-coded spiral diagram depicting polar motion between 1984 and 2010. The amplitude in the $x_{p}$ and $y_{p}$ components (horizontal scale) reaches 250 mas. Courtesy of S. Böhm.

\subsection{Troposphere and other ancillary products}

Observations of high-precision geodetic VLBI have been carried out for more than three decades providing a basis for the determination of geodynamic and astronomical parameters including their long-term variations in addition to the standard IVS products (TRF, CRF, and EOP) described above. For example, VLBI can determine Love numbers $h$ and $l$ of the solid Earth tides model (Spicakova et al., 2009), ionosphere models (Hobiger et al., 2006), or troposphere parameters. The long-term VLBI zenith wet delays are of interest for climatologists, because they contain information about the precipitable water vapor above the stations for their complete history (Heinkelmann et al., 2008). They can also be used to validate troposphere parameters from other space-geodetic techniques (Snajdrova et al., 2005; Teke et al., 2011). A typical example
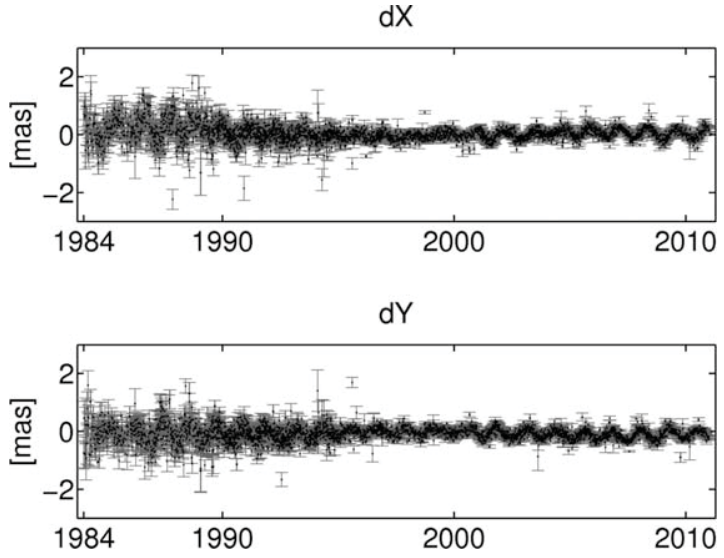

Fig. 10. $d X, d Y$ celestial pole offsets with respect to the IAU 2000/2006 precession-nutation model.

Courtesy of H. Spicakova, 2012. 


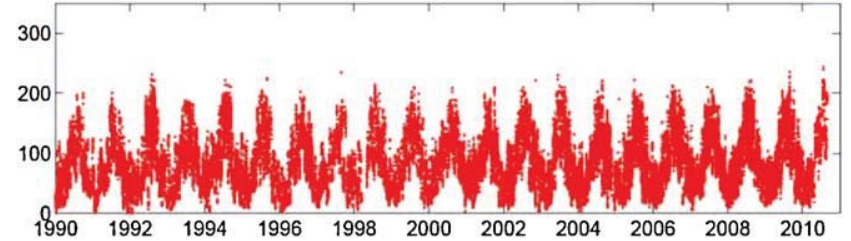

Fig. 11. Zenith wet delays (corresponding to precipitable water) above Wettzell from VLBI as obtained at IGG, Vienna University of Technology.

Courtesy of H. Spicakova, 2012.

for the variable zenith wet delay above a VLBI site is plotted for Wettzell station in Fig. 11.

Another interesting phenomenon, which can be observed by VLBI, is the gravitational deflection of radio waves by gravity according to general relativity. Radio waves are subject to space-time curvature caused by any massive body. In our solar system the largest effect occurs with radio waves which travel near the Sun. The effect of large planets such as Jupiter and Saturn is also appreciable. At an elongation angle of $2.5^{\circ}$ to the Sun, which was the minimal angle of VLBI observations until 2002, the differential deflection reaches 150 milliarcseconds (Robertson, 1991) causing a significant effect on the observed group delays. With respect to the noise floor of the source coordinates of about $40 \mu$ as for the ICRF2 (Fey et al., 2009), analysis of source observations in the vicinity of the Sun allows determining the post-Newtonian parameter $\gamma$ ('light deflection parameter'), which characterizes the space curvature due to gravity (see, e.g., Heinkelmann and Schuh, 2010). Although since 2002 the VLBI observations are scheduled with a minimal angle of $15^{\circ}$ to the Sun, the gravitational deflection still influences the measurements significantly and the most recent VLBI global solutions provided $\gamma$ with a precision of $1 \times 10^{-4}$ (Lambert and Le Poncin-Lafitte, 2009, 2011). The series of VLBI data is also sensitive to a possible acceleration of the solar system barycenter which might cause a secular drift of aberration with a magnitude of $4 \mu$ as/year (Sovers et al., 1998; Titov et al., 2011; Xu et al., 2012). Furthermore, the solar system motion relative to the cosmic microwave background might produce a dipole pattern that decreases with red shift (Titov et al., 2011).

\section{IVS, GGOS, and future perspectives of VLBI}

In the last 10-15 years, geodesy has experienced major changes with respect to its organizational structure. While up to the mid1990s most activities were organized in local, national, or regional frameworks, the last decade and a half has witnessed a shift to more global structures. On the initiative of the International Association of Geodesy (IAG), a number of technique-specific services were initially established, followed by an integrated observing system. Among the technique-specific services is the International VLBI Service for Geodesy and Astrometry (IVS); the overall "umbrella" system is the Global Geodetic Observing System (GGOS) of the IAG, which coordinates and integrates the geodetic activities on a global scale. In this section we describe the IVS as the technique service for VLBI and give a general introduction to the GGOS. We complete this section with an outline of future challenges for VLBI which originate from the scientific and user requirements for analyzing and modeling the system Earth.

\subsection{International VLBI Service for Geodesy and Astrometry}

The International VLBI Service for Geodesy and Astrometry (IVS) is an international collaboration of organizations which operate or support VLBI components. IVS was inaugurated on 1 March 1999 as a service of the IAG. In 2000, it was recognized as a service of the IAU and of the Federation of Astronomical and Geophysical Data Analysis Services (FAGS); the latter was dissolved in 2009 and replaced in 2011 by the World Data System (WDS).

IVS supports geodetic and astrometric work on reference systems and Earth science research in general, and provides the basis to all operational activities. According to the IVS Terms of Reference (see online at http://ivscc.gsfc.nasa.gov/ about/org/documents/ivsTOR.html) its mission objectives are:

1. to foster and carry out VLBI programs. This is accomplished through close coordination of the participating organizations to provide high-quality VLBI data and products;

2. to promote research and development activities in all aspects of the geodetic and astrometric VLBI technique;

3. to advance the education and training of VLBI participants through workshops, reports, and other means;

4. to support the integration of new components into IVS;

5. to interact with the community of users of VLBI products.

IVS is in charge of the integration of VLBI into a global Earth observing system and thus represents VLBI in the Global Geodetic Observing System (see below) of the IAG. IVS also interacts closely with the IERS, which is tasked by IAU and IUGG (International Union of Geodesy and Geophysics) with maintaining the reference frames ICRF and ITRF as described in Section 3.

To meet these objectives, IVS coordinates VLBI observing programs, sets performance standards for VLBI stations, establishes conventions for VLBI data formats and data products, issues recommendations for VLBI data analysis software, sets standards for VLBI analysis documentation, and institutes appropriate VLBI product delivery methods to ensure suitable product quality and timeliness. IVS also coordinates its activities with the astronomical community because of the dual use of many VLBI facilities and technologies for both radio astronomy and geodesy/astrometry.

VLBI data products currently available are the full set of Earth orientation parameters, the TRF, the CRF, and tropospheric parameters (see also Section 3). The IVS products can be defined in terms of their accuracy, reliability, frequency of observing sessions, temporal resolution of the estimated parameters, time delay from observing to final product, and frequency of solutions. Table 1 provides a current overview of the main products plus their specifications. All VLBI data and results in appropriate formats are archived in IVS Data Centers and are publicly available for research in related areas of geodesy, geophysics, and astrometry. The IVS data set extends from 1979.

The day-to-day activities as well as long-term plans of the IVS are coordinated from the central bureau of the service, called Coordinating Center, which is hosted by NASA's Goddard Space Flight Center in Greenbelt, MD, U.S.A. The IVS currently has about 80 permanent components supported by roughly 40 institutions in 20 countries. The permanent components and their functions within the IVS are:

- 30 Network Stations: acquiring high performance VLBI data;

- 3 Operation Centers: coordinating the activities of a network of network stations;

- 6 Correlators: processing the acquired data, providing feedback to the stations and providing processed data to analysts;

- 6 Data Centers: distributing products to users, providing storage and archiving functions;

- 26 Analysis Centers: analyzing the data and producing the results and products;

- 7 Technology Development Centers: developing new VLBI technology;

- 1 Coordinating Center: coordinating daily and long-term activities. 


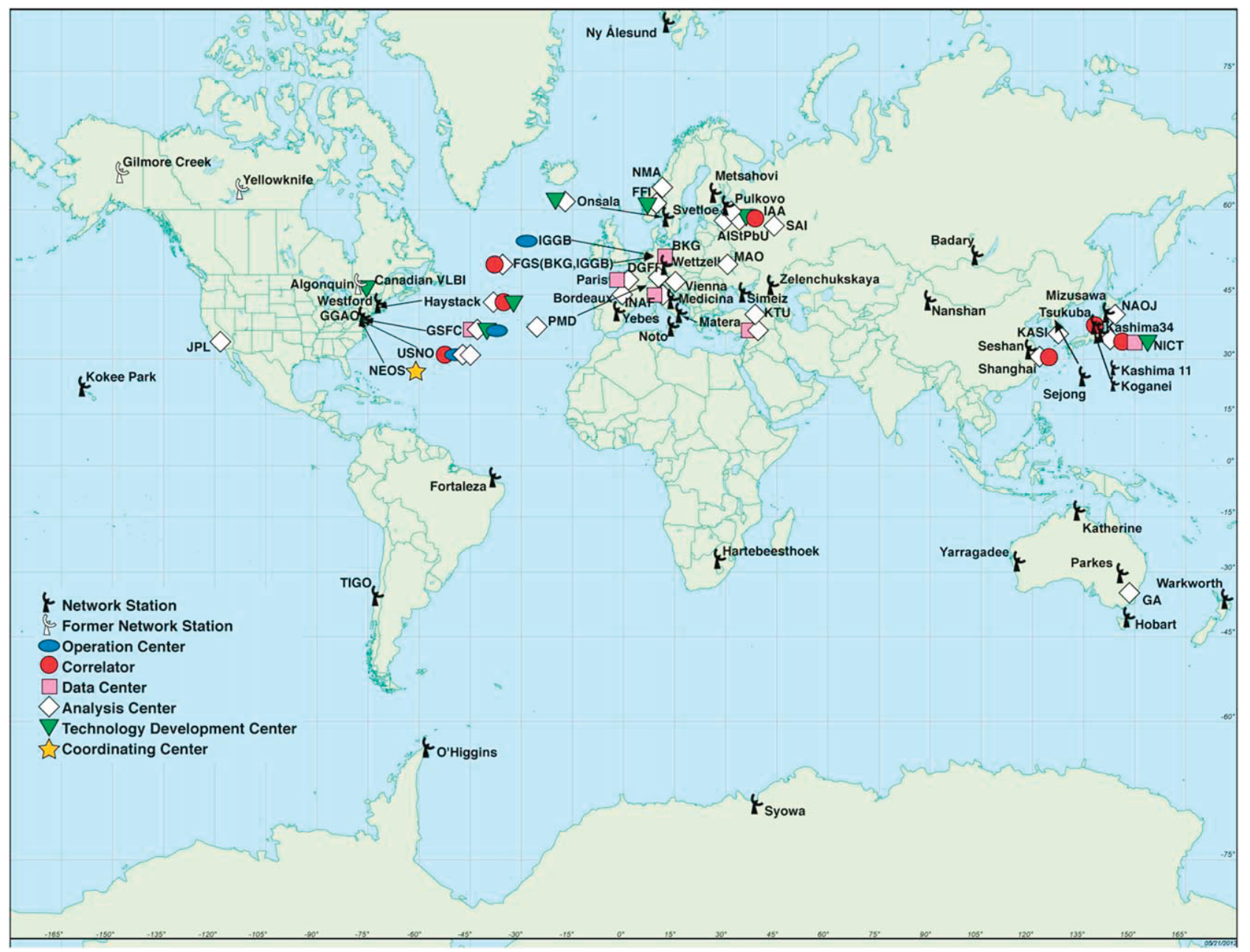

Fig. 12. Global distribution of IVS components.

The geographical distribution of the components has a bias for the northern hemisphere (see Fig. 12). While this can be partially explained by the fact that about two thirds of the land mass is located in the northern hemisphere, the paucity of components in the south has also geopolitical reasons. With the advent of the next generation VLBI system, the hope is that more components, in particular more Network Stations, can be established in Africa, South America, and in the southern hemisphere in general.

The observational network scheduled in the IVS observing plan comprises $\sim 30$ Network Stations plus $\sim 15$ stations from astronomical networks such as the VLBA (Very Large Baseline Array), the DSN (Deep Space Network), or the EVN (European VLBI Network), which cooperate with the IVS. A master observing plan is prepared for each calendar year based on the station availability time of the VLBI stations. Some stations carry a high load of observations and are included in most of the observing sessions, while other stations can only contribute to dedicated campaigns (Schlüter and Behrend, 2007). In 2011/2012, there are three-and-a-half 24-h sessions and seven daily 1 -h intensive sessions carried out per week. In other words, there are VLBI observations available for roughly $50 \%$ of the year. The actual observational networks consist of 8-10 stations in the case of the 24-h sessions and of 2-3 stations for the 1-h sessions. The daily Intensives are tailored for the determination of UT1-UTC only, whereas the 24-h sessions yield the full slew of parameters. More information about the IVS, its observing program and products, and other activities can be found online at http://ivscc.gsfc.nasa.gov.

\subsection{Global Geodetic Observing System}

Geodesy can be defined as the science that determines the geometry, gravity field, and rotation of the Earth, and their evolution in time (Plag and Pearlman, 2009). With the introduction of the space-geodetic techniques and the ensuing accuracy improvement of more than three orders of magnitude over the last three decades, geodesy has made unique contributions to the study of the Earth system, its inherent dynamics, and its response to climate change. It is also a tool underpinning a wide variety of other remote sensing techniques (Plag and Pearlman, 2009). Recognizing the need to have a common voice for the increasing number of technique-specific services as well as a link between IAG as a whole and relevant international Earth observation and research programs, the IAG initiated the GGOS during the IUGG General Assembly in 2003 (Plag et al., 2010). After an initial definition phase and the implementation of core elements of the organizational structure of GGOS, the IAG elevated the GGOS, at the IUGG General Assembly in 2007, to the status of permanent observing system of IAG (Plag et al., 2010). The official Web site with a wealth of information is available at http://www.ggos.org. The IAG, within the IUGG, contributes with the GGOS to the Global Earth Observing System of Systems (GEOSS). GEOSS is an outcome of the Group on Earth Observation (GEO) which is composed of 87 nations plus the European Commission and 64 participating organizations (as of January 2012). 


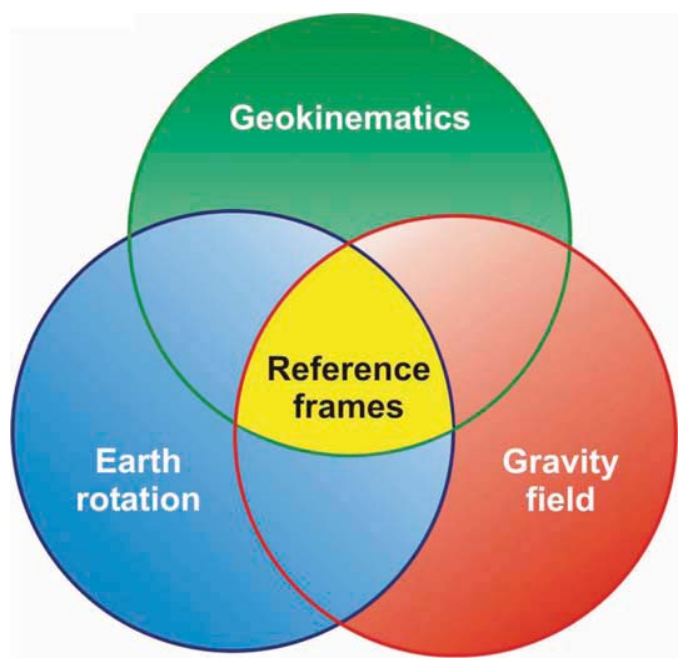

Fig. 13. Constituents of an integrated geodetic monitoring system. The three pillars of geodesy provide the conceptual and observational basis for the reference frames required for Earth observation.

Taken from Plag and Pearlman (2009), Fig. 1.1.

GGOS relies on the technique-specific services for providing the reference frames, which are crucial for Earth observing systems, and envisions the continued development of innovative technologies, methods, and models to improve our understanding of global change processes. GGOS provides a framework that ranges from the acquisition, transfer, and processing of a tremendous amount of observational data to its consistent integration and assimilation into complex numerical models of the Earth system (including solid Earth, oceans, atmosphere, hydrosphere, cryosphere, and the interactions thereof). In summary, GGOS provides essential contributions to an integrated Earth monitoring system to help us better understand global change and its impact on environment and society (Plag et al., 2009). The space-geodetic techniques and dedicated satellite missions are crucial for the determination and monitoring of geokinematics, Earth rotation and the gravity field (see Fig. 13). Together, these observations provide the basis to determine the geodetic reference frames with high accuracy, spatial resolution, and temporal stability. With the individual techniques contributing their particular strengths to the combination and integration, GGOS facilitates this process across the full parameter space as indicated in Table 3.

\section{Table 3}

Parameter space for a rigorous combination and integration of the geodetic observation techniques. Abbreviations used: GNSS (Global Navigation Satellite Systems), DORIS (Doppler Orbitography and Radiopositioning Integrated by Satellite), SLR (Satellite Laser Ranging), LLR (Lunar Laser Ranging). Adapted from Plag and Pearlman (2009), Table 9.1.

\begin{tabular}{lllllll}
\hline Parameter & VLBI & GNSS & DORIS & SLR & LLR & Altimetry \\
\hline ICRF (quasars) & $\mathrm{X}$ & & & & & \\
Nutation & $\mathrm{X}$ & $(\mathrm{X})$ & & $(\mathrm{X})$ & $\mathrm{X}$ & \\
Polar motion & $\mathrm{X}$ & $\mathrm{X}$ & $\mathrm{X}$ & $\mathrm{X}$ & $\mathrm{X}$ & \\
UT1 & $\mathrm{X}$ & & & & & \\
Length of day & $(\mathrm{X})$ & $\mathrm{X}$ & $\mathrm{X}$ & $\mathrm{X}$ & $\mathrm{X}$ & \\
ITRF (stations) & $\mathrm{X}$ & $\mathrm{X}$ & $\mathrm{X}$ & $\mathrm{X}$ & $\mathrm{X}$ & $(\mathrm{X})$ \\
Geocenter & & $\mathrm{X}$ & $\mathrm{X}$ & $\mathrm{X}$ & & $\mathrm{X}$ \\
Gravity field & & $\mathrm{X}$ & $\mathrm{X}$ & $\mathrm{X}$ & $(\mathrm{X})$ & $\mathrm{X}$ \\
Orbits & & $\mathrm{X}$ & $\mathrm{X}$ & $\mathrm{X}$ & $\mathrm{X}$ & $\mathrm{X}$ \\
LEO orbits & & $\mathrm{X}$ & $\mathrm{X}$ & $\mathrm{X}$ & & $\mathrm{X}$ \\
Ionosphere & $\mathrm{X}$ & $\mathrm{X}$ & $\mathrm{X}$ & & & $\mathrm{X}$ \\
Troposphere & $\mathrm{X}$ & $\mathrm{X}$ & $\mathrm{X}$ & & & $\mathrm{X}$ \\
Time/frequency & $(\mathrm{X})$ & $\mathrm{X}$ & & $(\mathrm{X})$ & & \\
\hline
\end{tabular}

The major outcome of GGOS in 2020 is expected to be a set of highly accurate, consistent, and long-term stable products which will be the geodetic contribution to the observation and monitoring of the Earth system. It is anticipated that all GGOS product accuracies in 2020 will be better than $10^{-9}$ relative to the absolute values of the measured quantities (Plag and Pearlman, 2009). This entails that the consistency between all GGOS products will be at the $10^{-9}$ level or better. As mentioned above, the IVS plays a key role within GGOS; thus, all IVS products are to be seen as GGOS products as well.

\subsection{Future challenges}

The quest for increasing accuracy, continuity, and timeliness of geodetic data as a benefit to both science and society has been at the root of the development of space-geodetic techniques from the very beginning some 40 years ago (Niell et al., 2006). The mounting number of natural disasters combined with the growth of the world population create an urgent need for mitigation measures such as prediction and warning systems. It has become mandatory to improve our understanding of the Earth system; for instance, a better understanding of sea level changes induced by global climate change will help mitigate the impact of these changes on the human population in the coastal regions. A necessary prerequisite for corresponding investigations is the availability and accessibility of very accurate global reference frames. Though being continually upgraded, the existing worldwide VLBI system has reached the limits of its capabilities and requires major renewal in order to provide the 1-mm accuracies demanded in the coming years (Niell et al., 2006).

Combining the scientific goals with the operational goals laid out in Schuh et al. (2002), the requirements for the next generation VLBI system can be essentially distilled into the following three distinct goals (e.g., Niell et al., 2006):

(1) accuracies of $1 \mathrm{~mm}$ in position and $1 \mathrm{~mm} /$ year in velocity;

(2) continuous measurement of station positions and Earth orientation parameters;

(3) turnaround time to initial products of less than $24 \mathrm{~h}$.

A realization of a new geodetic/astrometric VLBI system capable of fulfilling these goals seems feasible owing to technological developments over the past several years. These include the lower cost and faster antennas available today, advances in disk technology allowing high-data-rate recordings at an affordable cost, the availability of global optical-fiber networks, and advances in high-speed digital signal-processing technology.

\section{Next generation VLBI system}

In the first decade of this millennium the IVS established two working groups to define the outline of a next generation VLBI system, commonly known as the VLBI2010 system. The task of Working Group 2 "Product Specifications and Observing Programs" was to define the VLBI2010 measurement goals and to propose corresponding observing programs. The Working Group 2 report (Schuh et al., 2002) described the future demands of the service products. Several products, such as station coordinates, time series of baseline lengths and baseline components (including episodic events), Earth rotation velocity, rotational pole position, nutational parameters, as well as geophysical properties of the ionosphere and troposphere, demand continuous seven days per week observation. The follow-up IVS Working Group 3 “VLBI2010" was created in September 2003. It examined current and future requirements for VLBI geodetic systems, including all components from antenna 
to analysis, and published a final report with recommendations for a new generation of systems (Niell et al., 2006). The main characteristics of the future VLBI2010 system can be identified as follows:

- continuous observations in 30 s slew-track cycles,

- fast radio telescopes of $\leq 12-\mathrm{m}$ reflector class with kinematic parameters of either a single 12 -m diameter antenna with very high slew rates, e.g. $12 \% \mathrm{~s}$ in azimuth, or a pair of $12-\mathrm{m}$ diameter antennas, each with more moderate slew rates, e.g. $5 \%$ in azimuth (Petrachenko et al., 2009),

- wideband feed, $2-14 \mathrm{GHz}$ (later 2-18 GHz),

- digital baseband converter,

- high-data-rate sampling data acquisition, $\geq 8 \mathrm{Gbps}$,

- broadband connectivity for e-transfer and e-VLBI,

- distributed remote controlled continuous operation of the VLBI network,

- software correlator,

- automated production process including analysis.

The 1-mm position accuracy target postulated for the future VLBI system requires a delay measurement precision of $4 \mathrm{ps}$. This is nearly an order of magnitude improvement over current performance and cannot be achieved with existing dual-band S/X group delay systems (Petrachenko et al., 2009), which use dualband receivers with $\mathrm{S}$ band in the $2.2-2.4 \mathrm{GHz}$ range and $\mathrm{X}$ band in the $8.2-8.95 \mathrm{GHz}$ range. Further, the concept of the current VLBI system was conceived mostly some $30-40$ years ago. Notwithstanding the continuous efforts to improve the VLBI performance in terms of receiver technology and recording media, aging antennas, increasing radio frequency interference (RFI) problems, obsolete electronics, and high operating costs make it increasingly difficult to sustain the current levels of accuracy, reliability, and timeliness (Behrend et al., 2009). Recognizing these shortcomings, the IVS has been developing the VLBI2010 system which will replace the current $S / X$ system in the next several years.

The development of data acquisition systems for astronomy with more than $10 \mathrm{GHz}$ of instantaneous frequency coverage has opened up the possibility, for geodetic VLBI, of using multiple, widely spaced frequency bands to resolve the very precise radio frequency (RF) phase delay with only modest SNR per band. This has been demonstrated theoretically and allows the contemplation of systems that have excellent delay precision without the need for the high sensitivity that forces the use of large (and hence typically slowly moving) antennas. For an ideal operating environment with no RFI or source structure, it has been shown that a four-band system ( $1 \mathrm{GHz}$ per band) with RF frequency range $2-14 \mathrm{GHz}$ can reliably resolve phase delay with SNRs as low as 10 per band and achieve delay precision of $\sim 2$ ps (Petrachenko et al., 2009).

The development work has been underway since the abovementioned IVS Working Group on VLBI2010 (WG3) formulated and published its vision paper "VLBI2010: Current and Future Requirements for Geodetic VLBI Systems" (Niell et al., 2006). From 2006 on, implementation work has been done under the leadership of the IVS VLBI2010 Committee (V2C) encompassing theoretical studies and simulations, proof-of-concept investigations, and eventually hardware development and construction work. NASA is sponsoring a proof-of-concept development effort using VLBI antennas at Goddard Space Flight Center (12 m) and at MIT Haystack Observatory (the 18-m Westford antenna). Beyond the NASA development effort, organizations in other countries are involved in system developments potentially applicable to VLBI2010. These include Australia, China, Finland, Germany, Italy, Japan, Norway, Spain, Sweden, and others (Petrachenko et al., 2012).

Table 4 juxtaposes the main design features of the current and the next generation VLBI systems. With the new system the observations are to be taken by fast-slewing, 12-m class antennas (cf.
Table 4

Selected design features of the currently used legacy system and the next generation VLBI system (VLBI2010).

\begin{tabular}{lll}
\hline & Legacy system & VLBI2010 system \\
\hline Antenna size & $5-100 \mathrm{~m}$ dish & $\sim 12 \mathrm{~m}$ dish \\
Slew speed & $\sim 0.4-3^{\circ} / \mathrm{s}$ & $\sim 6-12^{\circ} / \mathrm{s}$ \\
Sensitivity & $200-15,000 \mathrm{SEFD}$ & $\leq 2500 \mathrm{SEFD}$ \\
Freq. range & $2.2-2.4 \mathrm{GHz}$, & Tunable over \\
& $8.2-8.95 \mathrm{GHz}$ & $\sim 2-14 \mathrm{GHz}$ \\
Freq. bands & 2 bands $(\mathrm{S}$ and $\mathrm{X})$ & 4 bands \\
Recording rate & $128-512 \mathrm{Mbps}$ & $8-32 \mathrm{Gbps}$ \\
Delay precision & $10-30 \mathrm{ps}$ & $\sim 4 \mathrm{ps}$ \\
Data transfer & Usually ship disks, & e-Transfer, e-VLBI, \\
& Some e-transfer & Ship disks as needed \\
\hline
\end{tabular}

Fig. 14) at a high data rate of $8 \mathrm{Gbps}$ and above. The implied higher sampling of the sky, as compared to the legacy system, will allow beating down the impact of error components. Together with a reduction of systematic errors, this will result in an anticipated overall accuracy of $1 \mathrm{~mm}$.

In order to shorten the on-source observing time, a new approach is being developed in which several widely spaced frequency bands are used to unambiguously resolve the interferometer phase (ambiguity-resolved phase delay). The new observable is being referred to as the broadband delay (BBD). To do this, a four-band system is recommended that uses a broadband feed to span the entire frequency range from 2 to $14 \mathrm{GHz}$. Fig. 15 shows the BBD system and the $\mathrm{S} / \mathrm{X}$ system in the frequency domain. In order to be able to detect an adequate number of radio sources, a total instantaneous data rate as high as $32 \mathrm{Gbps}$ and a sustained data storage or transmission rate of $8 \mathrm{Gbps}$ are necessary.

Fig. 16 describes the phase resolution process for the BBD. It is a multi-stage estimation process. Successively, the phase difference between the pair of bands with the smallest frequency separation is estimated and the corresponding integer cycles resolved until the phase is connected across the full frequency range. In a final step, the phase offset is determined. The system development effort for VLBI2010 involves nearly a complete reworking of the current S/X system (Petrachenko, 2010). The implications for the VLBI2010 signal path are profound. They include the use of linearly polarized broadband feeds, broadband low-noise amplifiers (LNAs), fiber-optic transmission of the RF signals from the antenna focus to the control room, an increase in the number of RF bands from two to four, and flexible frequency selection for each of the four RF bands (Petrachenko et al., 2009). A block diagram of the VLBI2010 system is shown in Fig. 17. Since the BBD technique is new

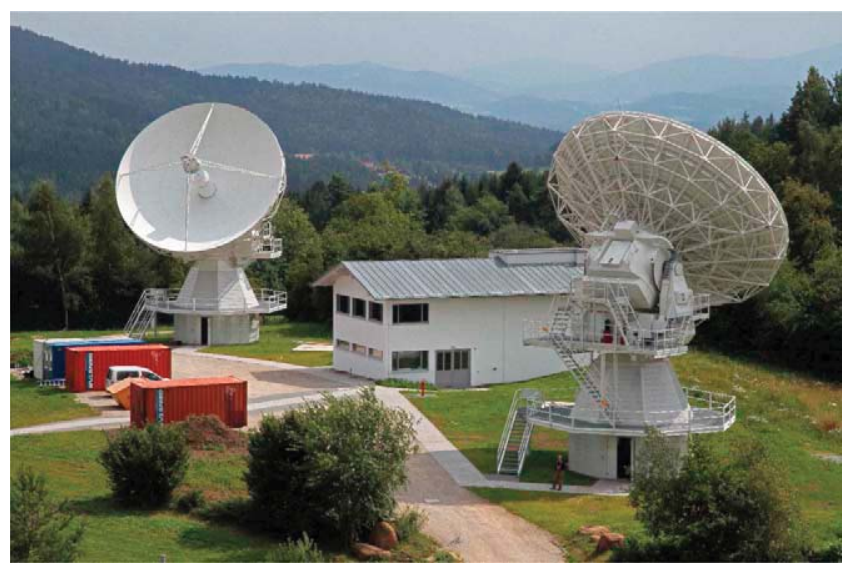

Fig. 14. Example of a VLBI2010-type antenna: the TWIN Telescope of the Geodetic Observatory Wettzell.

Photo courtesy of A. Neidhardt. 


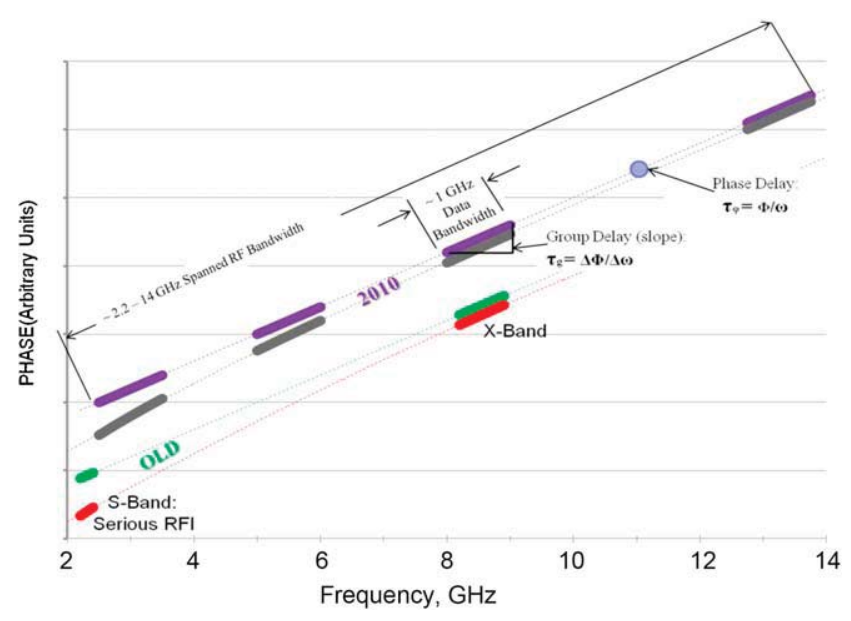

Fig. 15. Phase delay and group delay of the broadband system and the S/X system in the frequency domain.

Courtesy of T. Clark.

and untested, it is being verified in the proof-of-concept development effort mentioned above. The full progenitor VLBI2010 signal paths have been implemented on the 12-m GSFC antenna and the Westford antenna at Haystack Observatory. Fringes were detected using the full VLBI2010 broadband system at Goddard and a standard S/X feed and receiver with a VLBI2010 backend at Westford (Niell, 2011). Observations have started to evaluate the broadband performance.

The V2C undertook theoretical studies using Monte Carlo simulators in order to better understand the impacts of the new system and operating modes on final products. The simulators have been used to study the effects of the dominant VLBI random error processes (related to the atmosphere, the reference clocks, and the delay measurement noise) and the benefit of new approaches to reduce them, such as decreasing the source-switching interval and improving analysis and scheduling strategies (Petrachenko et al., 2012). Based on the results of these simulations, the requirements stemming from GGOS, redundancy and robustness considerations, and maintenance and repair requirements the V2C made the following recommendations for the minimum next generation VLBI network (Petrachenko et al., 2009):

- Have at least three regularly observing stations on each major tectonic plate, with more sites in regions where economics allow.

- Have at least eight regularly observing stations in the southern hemisphere.

- Have at least six regularly observing, globally distributed stations with high data rate connection to one or more correlators to enable near-real-time EOP delivery.

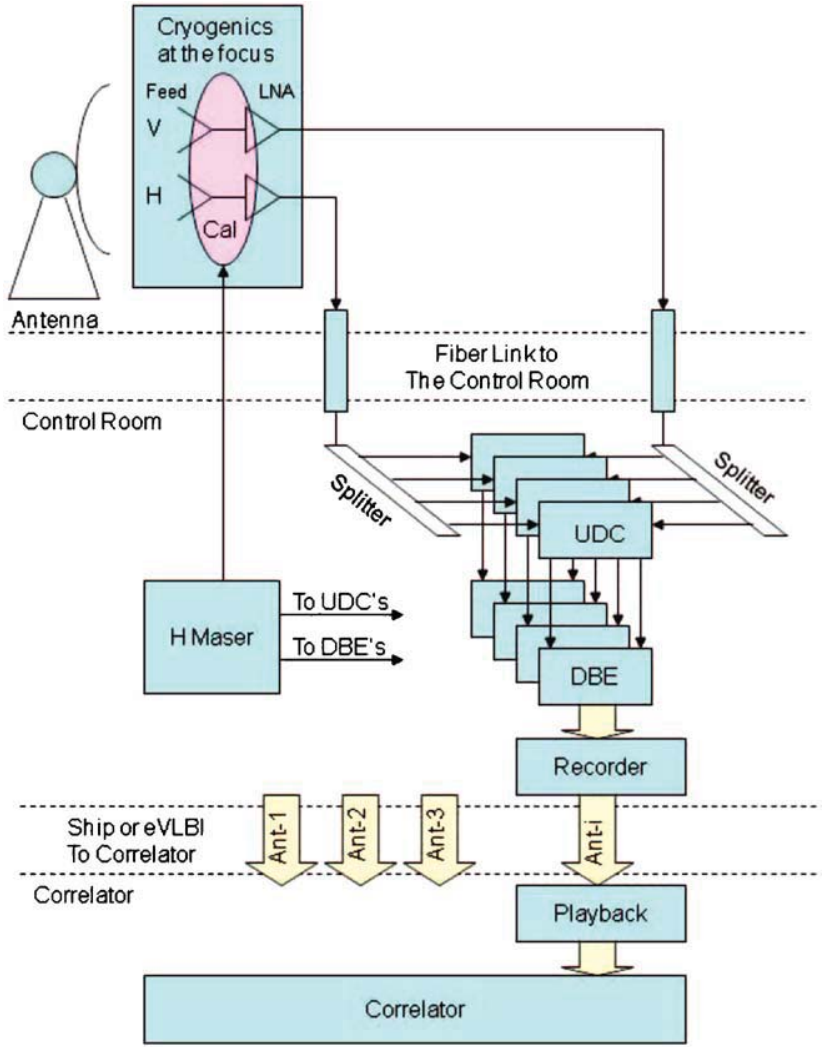

Fig. 17. VLBI2010 block diagram (Petrachenko et al., 2009). The front end at the antenna contains the broadband feed and Low Noise Amplifiers (LNAs), both cryogenically cooled to $\sim 20 \mathrm{~K}$ for increased sensitivity. The two senses of the linear polarization (vertical and horizontal) of the RF signals are used. The RF signals are brought down to the control room via optical-fiber links, where each RF band is frequency-translated to the intermediate frequency (IF) range in a flexible up-down converter (UDC). The output of the UDC is sampled at $10 \mathrm{bits} / \mathrm{sample}$ and processed in the digital back end (DBE) before being stored on disk recorder. A hydrogen maser provides the frequency and timing reference signals for the pulse calibration subsystem, the UDCs, and the DBEs. The recorded data are shipped or e-transferred to the correlator.

- Have at least eight larger (>20 m) antennas (four per hemisphere) for CRF densification.

- Wherever possible, co-locate new VLBI2010 stations near existing or planned space geodesy observatories, with a priority to SLR sites.

- Have a capability to process continuous observations for at least 24 stations, with a long-term goal to increase the number to at least 32 stations.

As of the beginning of 2012 a considerable number of VLBI2010 projects are underway; several antennas have been erected and construction of about ten more antennas world-wide is in various
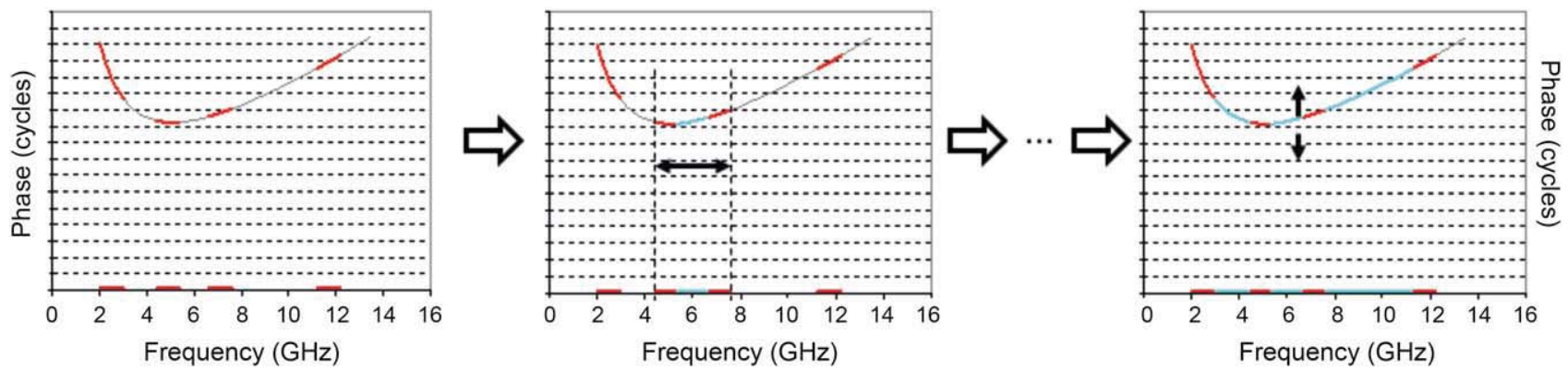

Fig. 16. Phase resolution for a given broadband sequence with $1 \mathrm{GHz}$ bandwidth. 
stages of completion. Further projects are in the proposal or planning stage. The next generation IVS network is growing, with an operational core of stations becoming available within the next few years, plus further growth continuing into the foreseeable future. A survey among the IVS Network Stations found that by 2013 a sufficient number of VLBI2010 compatible radio telescopes will be available for significant, but not full-time, VLBI2010 operations. By 2017, approximately 20 new radio telescopes at 17 sites will be available for VLBI2010 observations (Hase et al., 2011) and the IVS will get close to the desired 'seven days per week' operation, i.e. continuous monitoring of the EOP with a high temporal resolution. Additional new stations may also join if approved and constructed. However, even in 2017 the American/Pacific region will still lack the presence of VLBI2010 stations, though a 10-station NASA network covering some of this area may eventually be built (Hase et al., 2011).

\section{Conclusions and outlook}

The Very Long Baseline Interferometry (VLBI) technique has been employed for more than 30 years in geodesy, geophysics, and astronomy, and results of geodetic VLBI have been presented and interpreted in a multitude of publications by hundreds of authors. During the first two decades, most of the scientific and operational activities were organized through national or bi-lateral agreements only, which was an insufficiently strong basis for carrying out VLBI sessions in global networks. In 1999 the International VLBI Service for Geodesy and Astrometry (IVS) was established to coordinate the global VLBI components and resources on an international basis. It is an international collaboration of organizations that operate or support VLBI components for geodetic and astrometric applications. Specific goals are to provide a service to support geodetic, geophysical, and astrometric research and operational activities, to promote research and development activities in all aspects of the geodetic and astrometric VLBI technique, and to interact with the community of users of VLBI products and to integrate VLBI into a global Earth observing system. Since 2003 the Global Geodetic Observing System (GGOS; Plag and Pearlman, 2009) has been developed as a main component of the IAG, and the IVS provides an essential contribution to it.

In the last years the concept for the new generation VLBI system, VLBI2010, has been developed within the IVS and based on its specifications several new VLBI radio telescopes have already been approved worldwide. Thus, a highly capable VLBI2010 network will be implemented within this decade. New broadband 2-14 GHz observation modes will come into regular operation from $2014 / 2015$ onwards with full operation by about 2017 . The current $\mathrm{S} / \mathrm{X}$ operation mode will be maintained in parallel at a number of legacy stations for data continuity, astrometry, and space applications.

\section{Acknowledgements}

The continued success of VLBI as a space-geodetic technique would be impossible without the great support and enthusiasm from many institutions and individuals. This holds true in equal measure for the early years and for the last decade, during which VLBI has been done under the auspices of the IVS. The authors gratefully acknowledge the commitment made and resources provided by all IVS components. The authors would like to thank John Gipson and an anonymous reviewer for their useful comments to improve the manuscript. This paper is an outgrowth of the 2011 Vening Meinesz Medal speech of the first author at the European Geosciences Union (EGU) General Assembly in Vienna, Austria. The first author is grateful to the EGU and its Geodesy Section for awarding this medal and for recognizing the high significance that geodetic/astrometric VLBI has reached within the science community.

\section{References}

Altamimi, Z., Collilieux, X., Métivier, L., 2011. ITRF2008: an improved solution of the International Terrestrial Reference Frame. Journal of Geodesy, http://dx.doi.org/10.1007/s00190-011-0444-4(published online).

Böckmann, S., Artz, T., Nothnagel, A., 2010. VLBI terrestrial reference frame contributions to ITRF2008. Journal of Geodesy 84 (3), 201-219.

Behrend, D., Böhm, J., Charlot, P., Clark, T., Corey, B., Gipson, J., Haas, R., Koyama, Y., MacMillan, D., Malkin, Z., Niell, A., Nilsson, T., Petrachenko, B., Rogers, A., Tuccari, G., Wresnik, J., 2009. Recent Progress in the VLBI2010 Development. In: Sideris, M.G. (Ed.), Observing our Changing Earth, International Association of Geodesy Symposia, vol. 133. Springer-Verlag, Berlin, Heidelberg, pp. 833-840, ISBN: 978-3-540-85425-8.

Cannon, W., 1999. Overview of VLBI. In: Vandenberg, N., Baver, K. (Eds.), International VLBI Service for Geodesy and Astrometry 1999 Annual Report. NASA/TP-1999-209243. , pp. 13-17.

Fey, A., Gordon, D., Jacobs, C.S. (Eds.), 2009. The Second Realization of the International Celestial Reference Frame by Very Long Baseline Interferometry. IERS Technical Note 35. Verlag des Bundesamts für Kartographie und Geodäsie, Frankfurt am Main, ISBN: 3-89888-918-6, 204 pp.

Finkelstein, A.M., Kreinovich, V.J., Pandey, S.N., 1983. Relativistic reduction for radiointerferometric observables. Space Science 94, 233-247.

Hase, H., Behrend, D., Petrachenko, W., Schuh, H., Whitney, A., 2011. In: Alef, W., Bernhart, S., Nothnagel, A. (Eds.), Proc. 20th Meeting European VLBI Group for Geodesy and Astrometry, IGG Schriftenreihe 22. , pp. 78-81, ISSN: 1864-1113.

Heinkelmann, R., Schuh, H., 2010. Very long baseline interfrometry: accuracy limits and relativistic tests. In: Klioner, S.A., Seidelmann, P.K., Soffel, M.H. (Eds.), Proceedings of the International Astronomical Union Symposium 261 "Relativity in Fundamental Astronomy: Dynamics, Reference Frames, and Data Analysis"., pp. 286-290, ISSN: 1743-9213.

Heinkelmann, R., Böhm, J., Schuh, H., 2008. Very Long Baseline Interferometry (VLBI) for climate studies. In: Karslioglu, M.O., Nohutcu, M., Erdogan, E. (Eds.), Proceedings of the Turkish National Geodetic Commission Scientific Meeting 2007. , pp. 65-71.

Hobiger, T., Kondo, T., Schuh, H., 2006. Very long baseline interferometry as a tool to probe the ionosphere. Radio Science 41 (1), RS1006, http://dx.doi.org/10.1029/ 2005RS003297, 10 pp.

Lambert, S.B., Le Poncin-Lafitte, C., 2009. Determining the relativistic parameter $\gamma$ using very long baseline interferometry. Astronomy \& Astrophysics 499, 331-335.

Lambert, S.B., Le Poncin-Lafitte, C., 2011. Improved determination of $\gamma$ by VLBI. Astronomy \& Astrophysics 529, A70, http://dx.doi.org/10.1051/00046361/201016370, 4 pp.

Müller, J., 1991. Analyse von Lasermessungen zum Mond im Rahmen einer postNewton'schen Theorie. Verlag der Bayerischen Akademie der Wissenschaften, Deutsche Geodätische Kommission Reihe C383. ISBN: 3-7696-9430-9, 98 pp.

Ma, C., 1999. The celestial reference frame. In: Vandenberg, N., Baver, K. (Eds.), International VLBI Service for Geodesy and Astrometry 1999 Annual Report, NASA/TP-1999-209243. , pp. 18-22.

Ma, C., 2010. The second International Celestial Reference Frame (ICRF2). In: Behrend, D., Baver, K. (Eds.), International VLBI Service for Geodesy and Astrometry 2010 General Meeting Proceedings, NASA/CP-2010-215864. , pp. 273-279.

Niell, A., Whitney, A., Petrachenko, B., Schlüter, W., Vandenberg, N., Hase, H., Koyama, Y., Ma, C., Schuh, H., Tuccari, G., 2006. VLBI2010: current and future requirements of geodetic VLBI systems. In: Behrend, D., Baver, K. (Eds.), International VLBI Service for Geodesy and Astrometry 2005 Annual Report, NASA/TP-2006-214136. , pp. 13-40.

Niell, A., 2011. Status of the NASA VLBI2010 Proof-of-concept System. Presented at: 20th Meeting of the European VLBI Group for Geodesy and Astronomy, Bonn, Germany, 29-31 March 2011. http://www.mpifr-bonn.mpg.de/div/ meetings/20thEVGA/EVGA-Talks/Niell_VLBI2010_EVGA_110329.ppt.

Nilsson, T., Böhm, J., Böhm, S., Madzak, M., Nafisi, V., Plank, L., Spicakova, H., Sun, J., Tierno Ros, C., Schuh, H., 2011. Status and future plans for the Vienna VLBI software VieVS. In: Alef, W., Bernhart, S., Notnagel, A. (Eds.), Proceedings of the 20th Meeting of the European VLBI Group for Geodesy and Astronomy, Schriftenreihe des Instituts für Geodäsie und Geoinformation der Universität Bonn, Nr. 22. , pp. 93-96, ISSN: 1864-1113.

Petrachenko, B., Niell, A., Behrend, D., Corey, B., Böhm, J., Charlot, P., Collioud, A., Gipson, J., Haas, R., Hobiger, T., Koyama, Y., MacMillan, D., Malkin, Z., Nilsson, T., Pany, A., Tuccari, G., Whitney, A., Wresnik, J., 2009. Design Aspects of the VLBI2010 System - Progress Report of the IVS VLBI2010 Committee. NASA/TM2009-214180, 58 pp.

Petrachenko, W.T., Niell, A.E., Corey, B.E., Behrend, D., Schuh, H., Wresnik, J., 2012. VLBI2010: next generation VLBI system for geodesy and astrometry. In: Kenyon, S., Pacino, M.C., Marti, U. (Eds.), Geodesy for Planet Earth, International Association of Geodesy Symposia, vol. 136. Springer-Verlag, Berlin, Heidelberg, pp. 999-1006, ISBN: 978-3-642-20337-4.

Petrachenko, B., 2010. VLBI2010: an overview. In: Behrend, D., Baver, K. (Eds.), International VLBI Service for Geodesy and Astrometry 2010 General Meeting Proceedings, NASA/CP-2010-215864. , pp. 3-7. 
Plag, H.-P., Pearlman, M. (Eds.), 2009. Global Geodetic Observing System: Meeting the Requirements of a Global Society on a Changing Planet in 2020. SpringerVerlag, Berlin, Heidelberg, ISBN: 978-3-642-02686-7, 332 pp.

Plag, H.-P., Rothacher, M., Pearlman, M., Neilan, R., Ma, C., 2009. The global geodetic observing system. In: Satake, K. (Ed.), Advances in Geosciences, Vol. 13: Solid Earth. World Scientific Publishing Company, Singapore, pp. 105-128, ISBN: 9812836179.

Plag, H.-P., Rizos, C., Rothacher, M., Neilan, R., 2010. The Global Geodetic Observing System (GGOS): detecting the fingerprints of global change in geodetic quantities. In: Chuvieco, E., Li, J., Yang, X. (Eds.), Advances in Earth Observation of Global Change. Springer-Verlag, Berlin, Heidelberg, pp. 125-143, ISBN: 978-90481-9084-3.

Robertson, D.S., 1991. Geophysical applications of very-long-baseline interferometry. Reviews of Modern Physics 63 (4), 899-918.

Ryan, J.W., Ma, C., 1998. NASA-GSFC's geodetic VLBI program: a twenty-year retrospective. Physics and Chemistry of the Earth 23 (9-10), 1041-1052.

Schlüter, W., Behrend, D., 2007. The International VLBI Service for Geodesy and Astrometry (IVS): current capabilities and future prospects. Journal of Geodesy $81(6-8), 379-387$.

Schuh, H., Charlot, P., Hase, H., Himwich, E., Kingham, K., Klatt, C., Ma, C., Malkin, Z. Niell, A., Nothnagel, A., Schlüter, W., Takashima, K., Vandenberg, N., 2002. IVS Working Group 2 for product specification and observing programs. In: Vandenberg, N., Baver, K. (Eds.), International VLBI Service for Geodesy and Astrometry 2001 Annual Report, NASA/TP-2002-21000181. , pp. 12-45.
Seeber, G., 2003. Satellite Geodesy, 2nd ed. W. de Gruyter Verlag, Berlin, New York ISBN: 3-11-017549-5, 589 pp.

Shaffer, D.E., 1995. Geodetic measurements with VLBI. In: Zensus, J.A., Diamond, P.J., Napier, P.J. (Eds.), Very Long Baseline Interferometry and the VLBA. ASP Conference Series, vol. 82, pp. 345-361.

Snajdrova, K., Böhm, J., Willis, P., Haas, R., Schuh, H., 2005. Multi-technique comparison of tropospheric zenith delay derived during CONT02 campaign. Journal of Geodesy 79 (10-11), 613-623.

Sovers, O.J., Fanselow, J.L., Jacobs, C.S., 1998. Astrometry and geodesy with radio interferometry: experiments, models, results. Reviews of Modern Physics 70 (4), 1393-1454.

Spicakova, H., Böhm, J., Mendes Cerveira, P.J., Schuh, H., 2009. Determination of degree-2 Love and Shida numbers from VLBI. Bulletin d'Information des Marées Terrestres, 11679-11685, ISSN: 0542-6766.

Teke, K., Böhm, J., Nilsson, T., Schuh, H., Steigenberger, P., Dach, R., Heinkelmann, R. Willis, P., Haas, R., García-Espada, S., Hobiger, T., Ichikawa, R., Shimizu, S., 2011. Multi-technique comparison of troposphere zenith delays and gradients during CONT08. Journal of Geodesy 85 (7), 395-413.

Titov, O., Lambert, S.B., Gontier, A.-M., 2011. VLBI measurement of the secular aberration drift. Astronomy \& Astrophysics 529, A91, http://dx.doi.org/10.1051/00046361/201015718, 6 pp.

$\mathrm{Xu}$, M., Wang, G., Zhao, M., 2012. Direct estimation of the solar acceleration using geodetic/astrometric VLBI observations. Science China Physics, Mechanics \& Astronomy 55 (2), 329-332. 